\title{
BACKWARD AND FORWARD TRAVELLING WAVES IN EVOLUTIONARY GAMES *
}

\author{
V. HUTSON $^{\dagger}$ AND G. T. VICKERS ${ }^{\ddagger}$
}

\begin{abstract}
We consider a three strategy game in which a pure strategy is an evolutionarily stable strategy (ESS). The individuals are moving randomly (modelled by diffusion) in a (1-dimensional) spatial environment which is homogeneous. It is shown that, contrary to intuition, even if the ESS is spatially dominant against each of the other two strategies separately, there might be a travelling wave which will replace the ESS by one of them. The existence of the travelling wave is established by using a bifurcation technique.
\end{abstract}

1. Introduction. The incorporation of game theory into behavioural biology has proved to be extremely fruitful in the study of animal contests. The original formulation (for the biological context) is due to [15] and [14]. As proposed by these authors, there was no explicit inclusion of temporal or spatial effects. Temporal variation was first considered by [18] who proposed the replicator dynamic

$$
\frac{d u_{i}}{d t}=u_{i}\left[(A u)_{i}-u^{T} A u\right] \quad(1 \leq i \leq n) .
$$

In these equations, $A$ is the pay-off matrix, i.e. its entries give the pay-offs between the $n$ strategies. Also the vector $u$ gives the frequencies with which the $n$ strategies are being played. Each stable equilibrium point has its own basin of attraction and the union of their closures covers the whole of the unit simplex (an equilateral triangle for the three-strategy case considered here). The final outcome is thus easily determined by the initial conditions. Note that an ESS is necessarily asymptotically stable. Phase portraits for each of the topologically distinct cases are given by [21].

The fundamental idea behind an ESS is that it should be able to withstand invasion. Now it is to be expected that an attempted invasion will involve spatial and temporal variations in the frequencies with which the various strategies are being played. The simplest (and arguably the most natural) way of including spatial variation is via diffusion and this gives

$$
\frac{\partial u_{i}}{\partial t}=u_{i}\left[(A u)_{i}-u^{T} A u\right]+\frac{\partial^{2} u_{i}}{\partial x^{2}} \quad(1 \leq i \leq 3)
$$

This system of equations is the same as that proposed by [8] for the spatio-temporal variation in allele frequencies. Although [8] was only concerned with two alleles (or, in the present context, strategies) this work is particularly relevant because of its inclusion of travelling waves.

We do not concern ourselves with the underlying population dynamics. Rather the population density is presumed to be fixed. Of course, it is possible to incorporate a variable population density, but this will necessarily include new assumptions and may disguise the effect we wish to study. One advantage of extending the model in this way would be to allow the diffusion rates to be part of the strategy. That is not

\footnotetext{
${ }^{*}$ Received August 22, 2000; accepted for publication May 6, 2002.

${ }^{\dagger}$ Department of Applied Mathematics, The University of Sheffield, Sheffield, S3 7RH, U.K. (V.Hutson@sheffield.ac.uk).

${ }^{\ddagger}$ Department of Applied Mathematics, The University of Sheffield, Sheffield, S3 7RH, U.K. (g.vickers@sheffield.ac.uk).
} 
possible here. All individuals have the same diffusion rate which, for convenience, is taken as unity in equations (1.2).

Game theory was developed by [20] as a way of tackling problems in economics. The incorporation of spatial effects was rather late, perhaps because of the difficulty of modelling information flow in human situations. However, there are contexts where diffusion might be appropriate, and this is now receiving some attention. Here we mention [6], [10] and [11].

In classical ESS theory it is sufficient to consider different types of invaders one at a time. If a population is able to withstand invasion attempts by (a small number of) marauders who play a different strategy and if this same population can also repel marauders who play yet a different strategy, then it can withstand invasion by the two new types simultaneously. Suppose that in (1.2) we have

$$
A=\left[\begin{array}{ccc}
0 & -a_{12} & -a_{13} \\
-a_{21} & 0 & -a_{23} \\
-a_{31} & -a_{32} & 0
\end{array}\right]
$$

where $a_{13}>0$ and $a_{23}>0$ so that strategy 3 is an ESS. It has to be emphasised that invasion in the ESS context always refers to a small number of invaders: when there are two or more ESS's then of course one ESS can always be replaced by another if the number of invaders is sufficiently large. In the context of the system (1.2), it is reasonable to equate the success or otherwise of an invasion attempt by the directions of the travelling waves. For example, if $a_{31}>a_{13}>0$ then there will be a travelling wave, involving only strategies 1 and 3 , whose effect is to replace 3 by 1 . This wave could quite easily be generated in practise. If two sub-populations are temporarily isolated in neighbouring spatial regions, and each plays one of the strategies, then when contact is re-established it is very plausible that a wave will develop so as to remove one of the strategies. Such scenarios lead to the notion of dominance. This has been discussed by many authors including [7], [3] and [12], but here we follow [13]. Thus, a strategy is spatially dominant provided that any travelling wave has the result of leaving that strategy in its wake.

Suppose that the following conditions hold;

(a) $a_{13} \geq a_{31}>0$,

(b) $a_{23} \geq a_{32}>0$

with at least one of the inequalities (i.e. $\geq$ ) being strict. Not only is strategy 3 an ESS, but it dominates each of the strategies 1 and 2 in the sense that any travelling wave involving just 3 and 2 (or 3 and 1 ) has the effect of annihilating strategy 2 (or 1 ). So strategy 3 can repel invasion attempts by 1 and 2 separately. The above conditions are sufficiently strong to suggest that strategy 3 is spatially dominant: that is, the effect of any travelling wave will be to promote strategy 3. One of the main objectives of this paper is to show that this conjecture is false. Being able to defeat opponents one at a time is not sufficient. The effect of two simultaneous opponents (strategies 1 and 2) may be to defeat strategy 3. In a human context, this may not be surprising. It is easy to imagine opponents cooperating (perhaps temporarily) to defeat a third party; my enemy's enemy is my friend. This is not the case here. Each of the three strategies is operating for its own, short term, advantage. There is no sense of cooperation. It is to be stressed that this result is obtained without any 'extraneous' features. The diffusion rates of the strategies are all equal. The population size is fixed. These assumptions apply equally well in the classical situation. The conclusion has to be that classical ESS theory (and its extension (1.1)) is just not suited to 
answering the question 'can this population withstand invasion?'. Good alternative ways of answering this question are not easily found. If one has a full knowledge of the dynamics of the population (including how all different strategies interact) then, in principle, one studies (or numerically simulates) the model equations. Usually one does not have available such a perfect set of information. Indeed, perhaps the chief attraction of ESS theory was its ability to provide answers to questions about invasion when only a minimum amount of information was available.

As hinted earlier, our definition of spatial dominance is not the only one that has been considered. A natural alternative is to consider whether an isolated group, which play a particular strategy, can flourish. Specifically, one might say that strategy 3 is dominant if there exists a function $\bar{u}_{3}(x)$ of compact support such that if $u_{3}(x, 0) \geq$ $\bar{u}_{3}(x) \forall x$ then $\lim _{t \rightarrow \infty} u_{3}(x, t)=1$ uniformly on compact sets. It is tempting to believe that the two approaches are very similar, possibly identical, but we do not follow this further.

Using this idea of dominance, we shall show in section 6 that, although (a) and (b) are not sufficient for dominance, a strengthened condition may be found which is enough to ensure spatial dominance of a pure strategy.

The plan of the paper is as follows. After setting up the background in section 2 , in section 3 we give a formal argument suggesting a bifurcation from an edge wave. This depends critically upon our ability to obtain an unexpected, explicit solution of the variational equation. In section 4 we prove that under certain conditions a bifurcation does indeed take place by using the basic theorem of bifurcation for a simple eigenvalue. In section 5 we prove the assertion discussed above, that spatial dominance on the two edges does not imply dominance for the whole system. A sufficient condition for spatial dominance is given in the next section and finally, in section 7 , we give numerical results which demonstrate that the local bifurcation continues globally and we discuss some of the biological and economic consequences.

2. Background and notation. Here we present some basic facts about the model equations (1.2). It will be assumed throughout that

(H1): $a_{13}, a_{31}, a_{21}, a_{12}$ are positive.

The reaction terms of equations (1.2) are $u_{i} f_{i}$ where

$$
\begin{aligned}
f_{1} & =T-a_{12} u_{2}-a_{13} u_{3}, \\
f_{2} & =T-a_{21} u_{1}-a_{23} u_{3}, \\
f_{3} & =T-a_{31} u_{1}-a_{32} u_{2} \\
\text { and } T & =\left(a_{23}+a_{32}\right) u_{2} u_{3}+\left(a_{13}+a_{31}\right) u_{1} u_{3}+\left(a_{12}+a_{21}\right) u_{1} u_{2} .
\end{aligned}
$$

Let $\sigma=\sum_{i} u_{i}$. The addition of equations (1.2) gives

$$
\frac{\partial \sigma}{\partial t}=\frac{\partial^{2} \sigma}{\partial x^{2}}
$$

and the conclusion that the set $\sigma=1$ is invariant. Furthermore, if $u_{i}(x, 0) \geq 0 \forall i, x$, then $u_{i}(x, t) \geq 0 \forall i, x$ and $t>0$. Because the $u_{i}$ are interpreted as frequencies, it will always be assumed that this condition is true initially and so will remain true for all time.

Set $u_{2}=0$ in equations (1.2). Then $u_{3}=1-u_{1}$ and we have

$$
\frac{\partial u_{1}}{\partial t}=u_{1}\left(1-u_{1}\right)\left[\left(a_{13}+a_{31}\right) u_{1}-a_{13}\right]+\frac{\partial^{2} u_{1}}{\partial x^{2}} .
$$




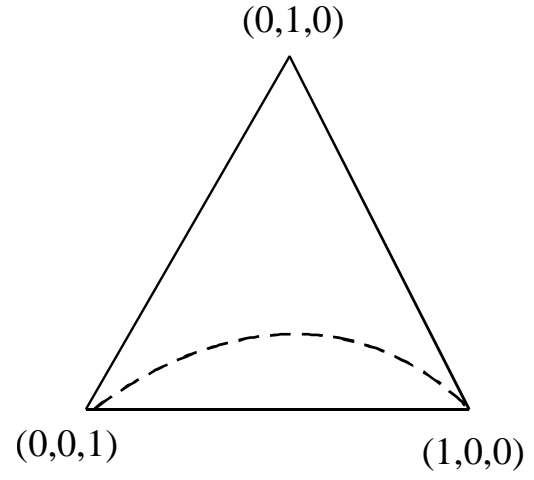

(a)

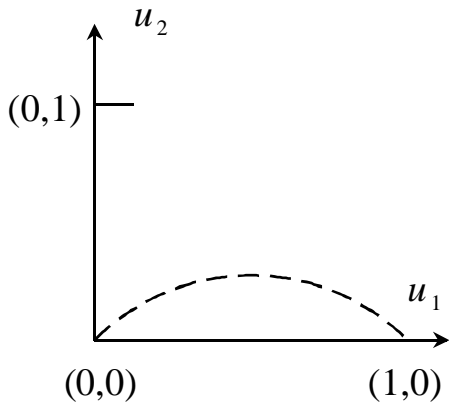

(b)

FIG. 3.1. Illustration of the elimination of $u_{3}$ using the relationship $u_{3}=1-u_{1}-u_{2}$. The broken line is the bifurcating travelling wave.

With the new independent variable $z=x-c t$, this becomes

$$
u_{1}^{\prime \prime}+c u_{1}^{\prime}+u_{1}\left(1-u_{1}\right)\left[\left(a_{13}+a_{31}\right) u_{1}-a_{13}\right]=0,
$$

where a prime denotes differentiation with respect to $z$, the travelling-wave variable. With the boundary conditions

$$
\lim _{z \rightarrow \infty} u_{1}=1 \text { and } \lim _{z \rightarrow-\infty} u_{1}=0
$$

the solution is $\left(\tilde{u}_{1}, \tilde{c}\right)$ where

$$
\tilde{u}_{1}(z)=\frac{1}{1+e^{-\gamma z}}, \quad \tilde{c}=\frac{a_{13}-a_{31}}{\sqrt{2\left(a_{13}+a_{31}\right)}} \text { where } \gamma=\sqrt{\frac{a_{13}+a_{31}}{2}} .
$$

We shall refer to this travelling-wave solution as an 'edge wave' or 'the 13 edge wave'. Clearly the direction of this wave is determined by the sign of $a_{13}-a_{31}$. Indeed, if $a_{13}>a_{31}$ then $\tilde{c}$ is positive and the effect of the wave is to replace strategy 1 by strategy 3 . Thus 3 is spatially dominant over 1 .

3. Bifurcation from an edge wave. A formal approach. Our aim is to show that in certain circumstances, the 13 edge wave loses stability to a travelling wave which is not an edge wave. This suggests there is a bifurcation. The notation is illustrated by Fig. 3.1, where, when $u_{3}$ is eliminated, there is a pair of partial differential equations in $u_{1}, u_{2}$. The tactics are to show that a travelling wave is generated in which $\lim _{x \rightarrow \pm \infty} u_{2}(x, t)=0$ (but $u_{2}(x, t)$ is not identically zero). The argument presented in this section is a formal one and we defer the question of proof until Section 4.

One may expect the stability, see Fig. 3.1, to be determined by the linearized $u_{2}$-equation. We shall throughout use the linearized variables

$$
u_{1}=\tilde{u}_{1}+v_{1}, \quad u_{2}=v_{2}, \quad c=\tilde{c}+\theta .
$$

Thus the linearized $u_{2}$-equation is

$$
\frac{\partial v_{2}}{\partial t}=v_{2} f_{2}\left(\tilde{u}_{1}, 0\right)+\frac{\partial^{2} v_{2}}{\partial x^{2}}
$$


where

$$
f_{2}\left(\tilde{u}_{1}, 0\right)=\left(a_{13}+a_{31}\right) \tilde{u}_{1}\left(1-\tilde{u}_{1}\right)-a_{21} \tilde{u}_{1}-a_{23}\left(1-\tilde{u}_{1}\right) .
$$

We are looking for a travelling-wave solution and so we consider a solution of equation (3.1) of the form $e^{\sigma t} V(z)$ where $z=x-c t$. Substitution of this into equation (3.1) yields

$$
V^{\prime \prime}+\tilde{c} V^{\prime}+f_{2} V=\sigma V
$$

where

$$
f_{2}=\frac{\gamma^{2}}{4}\left\{-\alpha-\beta \tanh \left(\frac{\gamma z}{2}\right)+2 \operatorname{sech}^{2}\left(\frac{\gamma z}{2}\right)\right\}
$$

Here

$$
\begin{aligned}
& \gamma^{2} \alpha=2\left(a_{21}+a_{23}\right) \\
& \gamma^{2} \beta=2\left(a_{21}-a_{23}\right)
\end{aligned}
$$

and it is convenient also to define $\Delta$ by

$$
\gamma^{2} \Delta=a_{13}-a_{31} .
$$

Rather surprisingly, one finds that equation (3.2) has a simple explicit solution. One can show, after some calculation, that the guess

$$
V(z)=\exp \left(\frac{a \gamma z}{2}\right) \operatorname{sech}\left(\frac{\gamma z}{2}\right)
$$

is a solution if

$$
\begin{array}{r}
a=-(\Delta+\beta) / 2, \\
a^{2}+1+a \Delta-\alpha=\frac{4 \sigma}{\gamma^{2}} .
\end{array}
$$

We impose the condition $|a|<1$ in order to ensure that $V \rightarrow 0$ as $|z| \rightarrow \infty$. Recalling the form of $v_{2}$, we see that the edge wave may be expected to lose stability to a travelling wave (which is not an edge wave) as $\sigma$ increases through zero. Calculation shows that this happens when the following pair of conditions holds:

$$
\begin{gathered}
-a_{31}<a_{23}-a_{21}<a_{13}, \\
a_{13} a_{31}-\left(a_{21}+a_{23}\right)\left(a_{13}+a_{31}\right)+\left(a_{21}-a_{23}\right)^{2}=0 .
\end{gathered}
$$

The above argument is suggestive, but not of course a proof that a bifurcation takes place.

4. Proof of a bifurcation for the case $\tilde{c}=0$. Our aim is to prove the existence of a travelling wave in the interior by using the technique associated with bifurcation from a simple eigenvalue. This method is described in [1] chapter 5 (and elsewhere). Although this technique has been much used in applications, its use appears to be relatively rare in the context of establishing the existence of a bistable travelling wave. A notable exception is the book [19] which discusses a variety of methods for travelling-wave problems. In the present case, the difficulties are much simplified by our knowledge of the explicit solution to equation (3.1) derived in the previous section. 
The bifurcation parameter is chosen to be $\lambda=a_{21}$. This is for convenience and does not imply any special property of this particular parameter. The basic travellingwave equations for $u_{1}(x-c t), u_{2}(x-c t)$ are

$$
\begin{aligned}
& u_{1}^{\prime \prime}+c u_{1}^{\prime}+u_{1} f_{1}\left(u_{1}, u_{2} ; \lambda\right)=0 \\
& u_{2}^{\prime \prime}+c u_{2}^{\prime}+u_{2} f_{2}\left(u_{1}, u_{2} ; \lambda\right)=0
\end{aligned}
$$

where a dash denotes differentiation with respect to $z=x-$ ct. We shall later need to consider the dynamical system generated and in preparation we note that this is derived from the following system

$$
\begin{aligned}
& u_{1}^{\prime}=p_{1} \\
& p_{1}^{\prime}=-c p_{1}-u_{1} f_{1} \\
& u_{2}^{\prime}=p_{2} \\
& p_{2}^{\prime}=-c p_{2}-u_{2} f_{2} .
\end{aligned}
$$

We shall assume throughout this section that the following conditions hold on the coefficients $a_{i j}$.

(H2): $a_{13}=a_{31}$,

(H3): $a_{23}<a_{13}$.

It follows from $(\mathrm{H} 2)$ that $\tilde{c}=0$. It is fairly easy to see that the conditions (3.5) and (3.6) (which ensure that the equation (3.2) has a solution which is exponentially small as $z \rightarrow \pm \infty$ ) are satisfied when (H2) and (H3) hold together with the condition $\lambda=\lambda_{0}$ where

$$
\sqrt{\lambda_{0}}=\gamma-\sqrt{a_{23}}
$$

As a preparation for setting up the equations in a form suitable for the application of the bifurcation theorem, we first shift the origin with the change of variable

$$
u_{1}=\tilde{u}_{1}+v_{1}, u_{2}=v_{2}, c=\theta .
$$

One notes that the wave speed $\theta$ is a part of the unknown solution. We shall write the problem in the form

$$
M(v, \lambda)=(B-\lambda D) v+N(v, \lambda)
$$

where with $X, Y$ Banach spaces, $B, D: X \rightarrow Y$ are linear operators and $N$ is an operator $X \rightarrow Y$. In fact the form of the problem suggests we take $X=B_{1} \times B_{2} \times \mathbb{R}$ and $y=B_{3} \times B_{4}$, where the $B_{i}$ are all Hilbert spaces (to be described shortly). We start with the linearization which leads to the matrix of operators $B$ and $D$ (formal for the moment) acting on the vector $\left(v_{2}, v_{2}, \theta\right)^{T}=v$. They are given by

$$
B=\left[\begin{array}{ccc}
B_{11} & B_{12} & B_{13} \\
0 & B_{22} & 0
\end{array}\right], \text { and } D=\left[\begin{array}{ccc}
0 & D_{12} & 0 \\
0 & D_{22} & 0
\end{array}\right] \text {, }
$$

where

$$
\begin{aligned}
B_{11} v_{1} & =v_{1}^{\prime \prime}+\gamma^{2}\left[6 \tilde{u}_{1}\left(1-\tilde{u}_{1}\right)-1\right] v_{1}, \\
B_{12} v_{2} & =\tilde{u}_{1}\left[\left(a_{12}-2 \gamma^{2}\right) \tilde{u}_{1}+\left(a_{23}+a_{32}\right)\left(1-\tilde{u}_{1}\right)-a_{12}+\gamma^{2}\right] v_{2}, \\
B_{13} \theta & =\tilde{u}_{1}^{\prime} \theta \\
B_{22} v_{2} & =v_{2}^{\prime \prime}+\left[2 \gamma^{2} \tilde{u}_{1}\left(1-\tilde{u}_{1}\right)-a_{23}\left(1-\tilde{u}_{1}\right)\right] v_{2}, \\
D_{12} v_{2} & =-\tilde{u}_{1}^{2} v_{2} \\
D_{22} v_{2} & =\tilde{u}_{1} v_{2} .
\end{aligned}
$$


It is also clear that $N(v, \lambda)$ is a polynomial in $v_{1}, v_{2}$ and that each term has degree 2 or 3 .

The above choice of space is dictated by the formal differential operators $B_{11}, B_{22}$. A crucial observation is that (by direct substitution)

$$
B_{11} \tilde{u}_{1}^{\prime}=0 .
$$

This is a consequence of a lack of uniqueness of the travelling wave $\tilde{u}_{1}$ (because of its invariance under a spatial translation), compare with [2] and [19]. In order to circumvent possible problems caused by this, we use a closed subspace of a certain Hilbert space which is orthogonal to the one-dimensional subspace generated by $\tilde{u}_{1}^{\prime}$.

Consider first the formal differential operators

$$
L_{1}=-B_{11}, \quad L_{2}=-\left(B_{22}-\lambda_{0} A_{22}\right),
$$

and write

$$
L_{i} v_{i}=-v_{i}^{\prime \prime}+g_{i} v_{i} .
$$

By equation (4.9), $L_{1} \phi_{1}=0$, where $\phi_{1}=\tilde{u}_{1}^{\prime}$. From the discussion in section 3 ,

$$
\phi_{2}=\exp \left(\frac{a \gamma z}{2}\right) \operatorname{sech}\left(\frac{\gamma z}{2}\right)
$$

satisfies $L_{2} \phi_{2}=0$. It is easy to check that in each case every other solution is unbounded for large $z$. It is straightforward to set up the problem with $L_{i}$ selfadjoint operators on the Hilbert space $H=L_{2}(\mathbb{R})$; for notational convenience we use the same symbol for the corresponding formal operators. A convenient reference for the standard theory is [5], chapter XIII. Let $\mathcal{D}(\cdot), \mathcal{R}(\cdot)$ and $\mathcal{N}(\cdot)$ denote the domain, range and kernel, respectively, of an operator. Let $A^{2}(\mathbb{R})$ denote the space of functions $f$ which have continuous derivatives and are such that $f^{\prime}$ is absolutely continuous. Let

$$
\mathcal{D}=\left\{f \in A^{2}(\mathbb{R}): f, f^{\prime}, f^{\prime \prime} \in H\right\},
$$

and take $\mathcal{D}\left(L_{i}\right)=\mathcal{D}$ for $i=1,2$. Then, [5] corollary XIII.6.15, the deficiency indices are $(0,0)$ and it follows that the $L_{i}$ are self-adjoint. Let $\sigma_{e}\left(L_{i}\right)$ denote the essential spectrum of $L_{i}$, see [5] page 1393. From [5] theorems XIII.7.4 (page 1438) and XIII.7.16(b) (page 1448) it follows that

$$
\sigma_{e}\left(L_{i}\right)=\left[\max \left(g_{i}^{+}, g_{i}^{-}\right), \infty\right),
$$

where $g_{i}^{ \pm}=\lim _{z \rightarrow \pm \infty} g_{i}(z)$ respectively. It is easy to check directly from the definition of the operators that

$$
g_{1}^{ \pm}=\gamma^{2}, \quad g_{2}^{+}=a_{21} \text { and } g_{2}^{-}=a_{23} .
$$

Therefore, from (H1), $0 \notin \sigma_{e}\left(L_{i}\right)(i=1,2)$. Hence $\mathcal{R}\left(L_{i}\right)$ is closed and $\mathcal{R}\left(L_{i}\right)=$ $\mathcal{N}\left(L_{i}\right)^{\perp}$. The above is summarized in the following lemma.

Lemma 4.1. The operators $L_{i}: \mathcal{D} \subset h \rightarrow H$ are self-adjoint. The kernel $\mathcal{N}\left(L_{i}\right)$ of each $L_{i}$ is one dimensional with basis $\phi_{i}$. Furthermore, $\mathcal{R}\left(L_{i}\right)$ is closed, $\mathcal{R}\left(L_{i}\right)=\mathcal{N}\left(L_{i}\right)^{\perp}$ and

$$
\mathcal{R}\left(L_{i}\right) \oplus \mathcal{N}\left(L_{i}\right)=H .
$$


Hence $L$ is Fredholm of index zero.

Denote the norm and inner product in $H$ by $\|\cdot\|$ and $(\cdot, \cdot)$, respectively. Take $H^{2}$ to be the Sobolev space $W^{2,2}(\mathbb{R})$ and let $\|\cdot\|_{H^{2}}$ and $(\cdot, \cdot)_{H^{2}}$ be the norm and inner product, respectively, in this space. Clearly, regarded as sets $\mathcal{D}=H^{2}$. In order to circumvent problems of non-uniqueness associated with the existence of an eigenfunction $\tilde{u}_{1}^{\prime}=\phi_{1}$ of $B_{11}$, see equation (4.9), we shall make use of the closed subspace $H_{0}^{2}$ of $H^{2}$ defined as follows:

$$
H_{0}^{2}=\left\{f \in H^{2}:\left(f, \phi_{1}\right)_{H^{2}}=0\right\} .
$$

With

$$
\begin{aligned}
& X=H_{0}^{2} \times H^{2} \times \mathbb{R}, \\
& Y=H \times H,
\end{aligned}
$$

we consider $M: X \rightarrow Y$. Then $B_{11}=L_{1}$ and $B_{22}-\lambda_{0} D_{22}=L_{2}$ are the differential operators whose properties are described in Lemma (4.1) and the remaining linear operators are simply multiplication operators. In the following, $V \in X$ will denote the non-trivial solution of

$$
\left(B-\lambda_{0} D\right) V=0
$$

we shall of course need to show that such a solution exists and is unique (up to multiplication by a constant).

TheOrem 4.2. Assume that (H1), (H2) and (H3) hold. Then $\left(\lambda_{0}, 0\right)$ is a bifurcation point of the equation $M(\lambda, v)=0$ and there exist analytic functions $\lambda^{\star}(\cdot)$ and $v^{\star}(\cdot)$ with

$$
\begin{aligned}
& \lambda^{\star}(s)=\lambda_{0}+O(|s|), \\
& v^{\star}(s)=s V+O\left(s^{2}\right),
\end{aligned}
$$

sufficiently near $s=0$, such that

$$
M\left(\lambda^{\star}(s), v^{\star}(s)\right)=0 .
$$

All solutions of $M(\lambda, v)=0$ near $\left(\lambda_{0}, 0\right)$ are either the trivial solution or are given by equations (4.12-4.13).

Proof. This is based on [1], Theorem 5.3, p. 189 together with the definition of a simple eigenvalue in section 5.3, p. 174. It is clear that the result follows from the following four properties:

1. $\operatorname{dim} \mathcal{N}\left(B-\lambda_{0} D\right)=1$.

We need first to prove the existence of the eigenvector $V$. From Lemma (4.1), $L_{2} \phi_{2}=0$, where $L_{2}=B_{22}-\lambda_{0} D_{22}$, and $\phi_{2}$ is a basis for $\mathcal{N}\left(L_{2}\right)$. With $V=\left(v_{1}, \phi_{2}, \theta\right),\left(B-\lambda_{0} D\right) V=0$ if and only if

$$
B_{11} v_{1}+\left(B_{12}-\lambda_{0} D_{12}\right) \phi_{2}+B_{13} \theta=0 .
$$

From Lemma (4.1), this equation has a solution if and only if

$$
\left(\left(B_{12}-\lambda_{0} D_{12}\right) \phi_{2}+B_{13} \theta, \phi_{1}\right)=0,
$$


that is

$$
\theta\left\|\phi_{1}\right\|^{2}+\left(\left(B_{12}-\lambda_{0} D_{12}\right) \phi_{2}, \phi_{1}\right)=0 .
$$

This equation obviously has a unique solution $\theta^{\star}$, say. Then from Lemma (4.1) again, equation (4.15) has family of solutions which, with $v_{1}$ a fixed solution of equation (4.15), may be expressed in the form

$$
v_{1}^{\star}=v_{1}+k \phi_{1} \quad(k \in \mathbb{R}) .
$$

However, we must have $v_{1}^{\star} \in H_{0}^{2}$, so that

$$
0=\left(v_{1}^{\star}, \phi_{1}\right)_{H^{2}}=\left(v_{1}, \phi_{1}\right)+k\left\|\phi_{1}\right\|_{H^{2}}^{2},
$$

which determines $k$. This yields a unique $v_{1}^{\star}$. This proves the first claim and gives the eigenfunction $V=\left(v_{1}^{\star}, \phi_{2}, \theta^{\star}\right)$ where $\theta^{\star}$ is determined by equation (4.16) and $v_{1}^{\star}$ by (4.15) and (4.17).

2. $\operatorname{codim} \mathcal{R}\left(B-\lambda_{0} D\right)=1$.

If $\left(g_{1}, g_{2}\right) \in \mathcal{R}\left(B-\lambda_{0} D\right)$ then

$$
\begin{aligned}
B_{11} v_{1}+\left(B_{12}-\lambda_{0} D_{12}\right) v_{2}+B_{13} \theta & =g_{1}, \\
B_{22} v_{2} & =g_{2} .
\end{aligned}
$$

From Lemma (4.1), equation (4.19) has a solution if and only if $\left(g_{2}, \phi_{2}\right)=0$ and then $v_{2}$ has the form $V_{2}+k_{2} \phi_{2}$ for some $V_{2}$ such that $B_{22} V_{2}=g$ and $k_{2} \in \mathbb{R}$. The use of Lemma (4.1) and a brief calculation shows that equation (4.18) has a solution if and only if

$$
\theta\left\|\phi_{1}\right\|^{2}=\left(g_{1}-\left(B_{12}-\lambda_{0} D_{12}\right) v_{2}, \phi_{1}\right) .
$$

A value of $\theta$ satisfying this equation may always be chosen and hence $\left(g_{1}, g_{2}\right) \in \mathcal{R}\left(B-\lambda_{0} D\right)$ if and only if $\left(g, \phi_{2}\right)=0$, that is, if and only if $\left(g_{1}, g_{2}\right) \perp\left(0, \phi_{2}\right)$ in the Hilbert space $H \times H$.

3. $D \mathcal{N}\left(B-\lambda_{0} D\right) \cap \mathcal{R}\left(B-\lambda_{0} D\right)=\{0\}$.

The vector $V$ is a basis for $\mathcal{N}\left(B-\lambda_{0} D\right)$. The second component $(D V)_{2}$ of $D V$ is $\tilde{u}_{1} \phi_{2}$. Hence, if the result is false, there must exist $v_{2} \in H^{2}$ such that

$$
\left(B_{0}-\lambda_{0} D_{22}\right) v_{2}=\tilde{u}_{1} \phi_{2} .
$$

By Lemma (4.1), this can only be the case if $\left(\tilde{u}_{1} \phi_{1}, \phi_{2}\right)=0$. This is impossible as $\tilde{u}_{1}, \phi_{1}, \phi_{2}$ are strictly positive functions.

4. The nonlinear operator $N$, see equation (4.8), is analytic.

As remarked previously, the non-linearities are polynomial. Hence the higher Fréchet derivatives are zero. The continuity of the map (and, by a similar argument, a finite number of its derivatives) follows from the continuous embedding of $H^{2}$ in $C^{\nu}$ for $0 \leq \nu<3 / 2$, see [9].

Theorem (4.2) shows that, for small $s$, there is a bifurcation solution

$$
\begin{aligned}
u_{1} & =\tilde{u}_{1}+s v_{1}^{\star}+s^{2} w_{1}, \\
u_{2} & =s \phi_{2}+s^{2} w_{2}, \\
c & =s \theta^{\star}+O\left(s^{2}\right), \\
\lambda & =\lambda_{0}+O(|s|),
\end{aligned}
$$


where

$$
\left\|w_{i}\right\|_{H^{2}} \leq k
$$

for some $k \in \mathbb{R}$. However, on its own, this result is not completely satisfactory. We wish to show that there is a travelling wave which satisfies the obvious biological constraints. Thus we require also that, with $p_{i}=u_{i}^{\prime}$ and $u=\left(u_{1}, p_{1}, u_{2}, p_{2}\right)$,

$$
u \rightarrow(0,0,0,0) \text { as } z \rightarrow-\infty \text { and } u \rightarrow(1,0,0,0) \text { as } z \rightarrow \infty .
$$

In addition, the range of $u_{1}, u_{2}$ must lie in $(0,1)$.

THEOREM 4.3. For small enough $s$, the bifurcation solution $\left(\tilde{u}_{1}, 0,0\right)+v^{\star}$ given by Theorem (4.2) is a travelling wave for the system (4.1), (4.2) satisfying (4.25). Furthermore, $0<u_{1}(z), u_{2}(z)<1$ for $z \in \mathbb{R}$.

Proof. As noted above, $H^{2}$ is continuously embedded in $C^{\nu}$ for $0 \leq \nu<3 / 2$, so it follows from equation (4.24) that for some $k \in \mathbb{R}$,

$$
\left\|w_{i}\right\|_{C^{\nu}} \leq k .
$$

To prove the first part of the result, it is enough to show that $u_{2}, u_{2}^{\prime} \rightarrow 0$ as $z \rightarrow \pm \infty$ (the proof for $u_{1}$ being analogous). We establish the result for $u_{2}^{\prime}$, the argument for $u_{2}$ being similar but slightly easier. In (4.26), take $\nu=1+\eta$ for some $\eta \in(0,1 / 2)$. Then for $z, z_{0} \in \mathbb{R}$,

$$
\left|u_{2}^{\prime}(z)-u_{2}^{\prime}\left(z_{0}\right)\right| \leq k\left|z-z_{0}\right|^{\eta} .
$$

If $u_{2}^{\prime} \nrightarrow 0$ then there exists $\epsilon>0$ and a sequence $\left\{z_{n}\right\} \rightarrow \infty$ such that either $u_{2}^{\prime}\left(z_{n}\right) \geq$ $2 \epsilon \forall n$ or $u_{2}^{\prime}\left(z_{n}\right) \leq-2 \epsilon \forall n$. Suppose, without loss of generality, that the first occurs. Then from equation (4.27),

$$
\begin{aligned}
u_{2}^{\prime}(z) & \geq u_{2}^{\prime}\left(z_{n}\right)-k\left|z-z_{n}\right|^{\eta}, \\
& \geq 2 \epsilon-k\left|z-z_{n}\right|^{\eta} .
\end{aligned}
$$

Therefore the right hand side is at least $\epsilon$ on an interval $I_{n}$ whose length $l$, say, is positive and independent of $n$. Thus $\int_{I_{n}} u_{n}^{\prime 2} d z \geq l \epsilon^{2}$ for all $n$. It follows that $u_{2}^{\prime} \notin L_{2}(\mathbb{R})$, which is a contradiction, since $u \in H^{2}$.

To prove the final assertion, we start by considering the dynamical system in $\mathbb{R}^{4}$ generated by equations (4.3)-(4.6), with $u=\left(u_{1}, p_{1}, u_{2}, p_{2}\right)$, and look for a connection of the hyperbolic equilibrium points $P(0,0,0,0)$ and $P(1,0,0,0)$. We have just shown that indeed we have such a connection, with the orbit leaving $P_{1}$ along the unstable manifold and approaching $P_{2}$ along the stable manifold. A linear analysis at each of $P_{1}$ and $P_{2}$ shows that the linear stable and unstable manifolds satisfy the required conditions. Using a strong version of the Stable Manifold Theorem, [17], we can conclude that there is $\delta>0$ and $s_{0}>0$ such that for $|s|<s_{0}$ the orbit is close enough to the linear unstable manifold to ensure that the required condition holds in the open ball $B\left(P_{1}, \delta\right)$ centre $P$ and radius $\delta$. The argument is similar near $P_{2}$.

We must show that the condition holds on the rest of the orbit. Choose $s_{1} \in$ $\left(0, s_{0}\right)$ such that

$$
s_{1}^{2}\left\{\left\|v_{1}^{\star}\right\|_{C^{1}}^{2}+\left\|\phi_{2}\right\|_{C^{1}}^{2}+\left\|w_{1}\right\|_{C^{1}}^{2}+\left\|w_{2}\right\|_{C^{1}}^{2}\right\} \leq \delta^{2} / 4 .
$$


Assume that the maximum of $\phi_{2}$ occurs at $z=0$ (by a change of variable, if necessary). Define $z_{1}$ by requiring that $\tilde{u}_{1}\left(z_{1}\right)=\delta / 2$. Then $u(z) \in B\left(P_{1}, \delta\right)$ for $z \leq z_{1}$ for all $s \in\left[0, s_{1}\right]$ and, if $z_{0}$ is the smallest $z$ with $u\left(z_{0}\right) \in \partial B\left(P_{1}, \delta\right)$, then $z_{0} \geq z_{1}$. Since $\phi_{2}(z)$ is increasing on $(-\infty, 0)$,

$$
\phi_{2}(z)>\phi_{2}\left(z_{1}\right) \quad\left(z \in\left(z_{0}, 0\right)\right) .
$$

We now choose $s_{2} \in\left(0, s_{1}\right)$ such that $s_{2}\left\|w_{2}\right\|_{C^{1}} \leq \frac{1}{2} \phi_{2}\left(z_{1}\right)$. Then $u_{2}(z)>0$ on $\left[z_{0}, 0\right]$. Clearly the argument also give $0<u_{1}(z)$ on $\left[z_{0}, 0\right]$ and $u_{1}(z)<1$ on $[0, \infty)$.

The proof of the existence of a bifurcating wave has been restricted for simplicity to the case when $\tilde{c}=0$, the self-adjointness of the ordinary differential operator making for a reduction of the technicalities. However, we remark that, with some extra effort, it is possible to obtain an analogue of the key result Lemma(4.1), with suitable amendments to incorporate the adjoint operators. The proof of Theorem (4.2) then goes through with minor changes. Since our main aim is to obtain a counterexample to the conjecture discussed in the introduction, we are content to give only the details of the proof for the special case. However, there is every reason to believe that the pair of conditions (3.3) and (3.4) give the condition for a bifurcation in the general case.

5. The Conjecture. We have suggested in the introduction that it is extremely plausible to conjecture that if $u_{3}$ is spatially dominant over $u_{1}$ when $u_{2}$ is absent and over $u_{2}$ in the absence of $u_{1}$ (that is, on each of the 1-3 and 1-2 edges) then it is spatially dominant for the full system. We shall show that in fact this conjecture is incorrect (some biological consequences are discussed later). This is done by using the travelling wave constructed in the last section and showing that its direction may be chosen such that $(1,0,0)$ is the winner - that is the component $u_{1}(x-c t) \rightarrow 1$ as $t \rightarrow \infty$, which happens if $c<0$ where we are assuming that $u_{1}(\cdot) \nearrow$.

We assume the following restrictions on the coefficients:

(i) $a_{13}=a_{31}$,

(ii) $a_{23}>a_{32}$.

Thus there is a standing wave on the 1-3 edge and a wave towards $(0,0,1)$ (i.e. with $u_{3}$ the winner) on the 2-3 edge. We have also assumed, see (H3), that $a_{23}<a_{31}$. Choose now

(iii) $a_{23}=a_{21}$.

In order to find the wave speed of the bifurcating wave, we use equation (4.16) to determine $\theta^{\star}$. All the terms in this equation are known explicitly with $\phi_{1}=\tilde{u}_{1}^{\prime}$ and $\phi_{2}$ given by equation (4.10). The evaluation of the inner product in equation (4.16) involves an integration over $\mathbb{R}$, and a great deal of tedious detail is avoided by using Maple. It is found that

$$
\theta^{\star}=\frac{9 \pi}{32 \gamma}\left(a_{12}-a_{32}\right) .
$$

Combining this with equation (4.22), we see that $c$ is negative when $a_{12}<a_{32}$. This yields the required wave speed and gives us our contradiction.

We remark that in fact a stronger result is almost certainly valid. If $a_{13}>a_{31}$ then as mentioned at the end of the last section, the existence of a bifurcating wave still holds. Proceeding now as before, we may choose $s>0$ in equation (4.22) to obtain a negative value for $c$. Now make a small increase in $a_{13}$ keeping $a_{31}$ fixed (or vice versa). Then, if $c$ is a continuous function of the parameter $a_{13}$, we will 
have opposite directions for the edge wave and for the bifurcating wave. This would prove that even the weaker conjecture, that is $a_{13}>a_{31}, a_{23}>a_{32}$ implies spatial dominance, is false. However, we are not aware that there is a satisfactory proof of the required continuity in $c$.

6. A Sufficient Condition for Spatial Dominance. We have shown in the previous section that, somewhat contrary to intuition, strategy 3 being dominant to each of strategies 1 and 2 separately is not sufficient to ensure that it is spatially dominant (i.e. a travelling wave may exist which removes strategy 3 ). One might raise then raise the question as to whether there are conditions which $d o$ ensure dominance. We shall show that in fact a relatively mild strengthening of the conditions (a) and (b) in section 1 will correct the deficiency. The result is given in terms of the alternative approach to dominance mentioned in the introduction. That is, we show that there is a function $\Phi(x)$ of compact support such that if $u_{3}(x, 0) \geq \Phi(x) \forall x$ then $u_{3}(x, t) \rightarrow 1$ uniformly on compact sets as $t \rightarrow \infty$.

Suppose throughout this section that condition (H4) below is imposed.

(H4): $a_{i j}>0(\forall i, j, i \neq j)$ and one of the following conditions holds:
(i) $a_{23}>a_{13}>a_{31}>a_{32}$,
$\left(i^{\prime}\right) a_{13}>a_{23}>a_{32}>a_{31}$,
(ii) $a_{23}>a_{13}>a_{32}>a_{31}$,
$\left(i i^{\prime}\right) a_{13}>a_{23}>a_{31}>a_{32}$.

Lemma 6.1. Assume that (H4) holds for some fixed set of parameters $a_{i j}$. Then there exist constants $A, B$ with $0<2 B<A$ such that

$$
f_{3}\left(u_{1}, u_{2}, u_{3}\right) \geq f\left(u_{3}\right)
$$

for all $u \in \mathbb{R}^{3}$ with $u_{1}, u_{2}, u_{3} \geq 0$ and $u_{1}+u_{2}+u_{3}=1$, where $f(u)=u(1-u)(A u-B)$.

Proof. Eliminating $u_{2}$ from the expression for $f_{3}$ by using $u_{1}+u_{2}+u_{3}=1$, we readily deduce the inequality

$f_{3} \geq u_{3}\left\{u_{1}\left[\left(a_{31}+a_{13}\right) u_{3}-a_{31}\right]-u_{1}\left[\left(a_{32}+a_{23}\right) u_{3}-a_{32}\right]+\left(1-u_{3}\right)\left[\left(a_{32}+a_{23}\right] u_{3}-a_{32}\right\}\right.$.

As $0 \leq u_{1} \leq 1-u_{3}$ and the right-hand side is a linear function of $u_{1}$, it cannot be smaller than the minimum of its values at the end points $u_{1}=0, u_{1}=1-u_{3}$. Therefore

$$
f_{3} \geq u_{3}\left(1-u_{3}\right) \min \left\{\left[\left(a_{31}+a_{13}\right) u_{3}-a_{31}\right],\left[\left(a_{32}+a_{23}\right) u_{3}-a_{32}\right]\right\} .
$$

We deduce the result from a simple geometrical argument based on the observation that the graph of each term within the min is a straight line. The graphs join the points $\left(0,-a_{31}\right)$ to $\left(1, a_{13}\right)$ and $\left(0,-a_{32}\right)$ to $\left(1, a_{23}\right)$. In case $(i),-a_{32}>-a_{31}$ and $a_{13}<a_{23}$. Thus the first line lies below the second and

$$
\left.f_{3} \geq u_{3}\left(1-u_{3}\right)\left[\left(a_{31}+a_{13}\right) u_{3}-a_{31}\right]\right\} .
$$

But $a_{13}>a_{31}$ and the result follows with $A=a_{31}+a_{13}, B=a_{31}$. In case (ii), the lines cross but both of them lie above the line joining $\left(0,-a_{32}\right)$ to $\left(1, a_{13}\right)$. Hence

$$
f_{3} \geq u_{3}\left(1-u_{3}\right)\left[\left(a_{32}+a_{13}\right) u_{3}-a_{32}\right] .
$$

Since $a_{13}>a_{32}$, the result follows with $A=a_{32}+a_{13}, B=a_{32}$.

The scalar case

$$
v_{t}=\mu v_{x x}+f(v),
$$


with $f$ as in the lemma, is extremely well known and the tactics will be to use (6.1) together with a comparison theorem for the $u_{3}$ component. The required result is summarised in Lemma 6.2 below. From Lemma 6.1, there is an $\alpha \in(0,1)$ with $\int_{0}^{\alpha} f(s) d s>0$; choose any such $\alpha$ and define $\phi$ to be the solution of

$$
\frac{d^{2} \phi}{d x^{2}}+f(\phi)=0 \quad\left(\phi(0)=\alpha, \phi^{\prime}(0)=0\right)
$$

on the maximal interval $\left(-x_{0}, x_{0}\right)$ on which $\phi$ remains positive. Set

$$
\Phi(x)= \begin{cases}\phi(x) & \left(|x|<x_{0}\right) \\ 0 & \left(|x| \geq x_{0}\right)\end{cases}
$$

LEMma 6.2. [7] Let $f$ be as in Lemma 6.1 and suppose that $v$ satisfies (6.2). Assume that $v(x, 0)$ is uniformly continuous and

$$
\Phi(x) \leq v(x, 0) \leq 1
$$

Then $v(x, t) \geq \Phi(x)(x \in \mathbb{R}, t>0)$ and $v(x, t) \rightarrow 1$ as $t \rightarrow \infty$ uniformly on compact sets.

Consider the initial value problem for the system (1.2) with

$$
\sum_{i=1}^{3} u_{i}(x, 0)=1 \quad \text { and } \quad u_{i}(x, 0) \geq 0 \forall i, x \in \mathbb{R} .
$$

As remarked earlier, these conditions ensure that for $x \in \mathbb{R}, t>0$ both $\sum_{i} u_{i}(x, t)=1$ and $u_{i}(x, t) \geq 0 \forall i$. This gives an easy a priori bound on the solutions and a standard existence theorem [4] ensures that we have a semiflow on the Banach space $B U C(\mathbb{R})$ of bounded, uniformly continuous functions on $\mathbb{R}$. The result on dominance may now be stated as follows.

THEOREM 6.3. Assume that (H4) holds and consider the initial value problem described above with $u_{i}(\cdot, 0) \in B U C(\mathbb{R})$ for each $i$. Suppose that $u_{3}(x, 0) \geq \Phi(x)(x \in$ $\mathbb{R})$. Then $u_{3}(x, t)>\Phi(x)$ for $t>0$ and $u_{3}(x, t) \rightarrow 1$ as $t \rightarrow \infty$ uniformly on compact sets.

Proof. For a given set of initial values, we set $u=u_{3}$ and $f_{3}\left(u_{1}(x, t), u_{2}(x, t), u\right)=$ $f(u, x, t)$. Then $u$ satisfies the equation

$$
\frac{\partial u}{\partial t}=\mu \frac{\partial^{2} u}{\partial x^{2}}+f(u, x, t)
$$

Furthermore, from Lemma $6.1, f(u, x, t) \geq f(u)$. We now use a standard comparison argument. Put $w=u-v$ where $v$ satisfies (6.2). Then from (6.2) and (6.3)

$$
\begin{aligned}
\frac{\partial w}{\partial t}-\mu \frac{\partial^{2} w}{\partial x^{2}} & =f(u, x, t)-f(v) \\
& \geq f(u)-f(v) \\
& =w \frac{f(u)-f(v)}{u-v} \quad(u \neq v) .
\end{aligned}
$$

As $f$ is smooth (in fact a cubic), $[f(u)-f(v)] /(u-v)$ is smooth and bounded. Also, from the maximum principle on $\mathbb{R}$, (see e.g. [16] chapter 3 theorem 10) $w \geq 0$. The result follows from Lemma 6.2. 
One may remark that other criteria for dominance may be obtained. For example, one can obtain an alternative to (H4) by examining other choices for $f$ in Lemma 6.1 and requiring that $\int_{0}^{1} f(s) d s>0$. Another possibility is to impose a condition which ensures that there is a supersolution for the $u_{1}$ equation which is unity except on a compact set. An argument similar to the above may then be used to show that $u_{1} \rightarrow 0$ uniformly on compact sets and hence, with some extra analysis, obtain a slightly different form of dominance for strategy 3. However, in view of our previous results, it is likely to be extremely hard to find a necessary and sufficient condition for dominance and we do not pursue this further.

7. Numerical results and discussion. Let $A$ be given by

$$
A=-\left[\begin{array}{ccc}
0 & 1.5 & 12.5 \alpha \\
3 & 0 & 2 \\
12 \alpha & 1 & 0
\end{array}\right]
$$

where $\alpha>0$. Each of the 13 and 23 edge waves promotes strategy 3 . It may be shown from (3.5) and (3.6) that when $\alpha \approx 0.81$ there is a bifurcation in the 13 edge wave. For $\alpha$ 'close' to (and larger than) this value, the direction of the new wave is in the same sense as the edge wave, i.e. its effect is also to promote strategy 3 . However, if $\alpha$ is increased significantly then the direction of the wave is reversed. Figure 7.1 shows the results when $\alpha=1.2$ (where already the wave favours strategy 1 ) and $\alpha=4$. This demonstrates the global nature of the bifurcation. As $\alpha$ is increased, the projection of the wave onto the triangle of reference approaches the edges of the triangle. However, the speed of the interior wave is quite different from either of the speeds of the edge waves and so, as a trajectory in $\mathbb{R}^{4}$, the interior wave is not close to either edge wave. It is noteworthy that the numerical results support the contention that the wave speed approaches a finite limit $(\approx 0.0435)$ as $\alpha$ is increased indefinitely. Now it is the sign of $c$ which determines the direction of the wave and hence the winning strategy. Thus it would be valuable to be able to give the general condition under which the wave speed is zero, as this would determine the marginal cases. Although we are not able to give such conditions, a heuristic argument is presented in the Appendix to show that, for the matrix

$$
A=-\left[\begin{array}{ccc}
0 & a_{12} & \alpha a_{13} \\
a_{21} & 0 & a_{23} \\
\alpha a_{31} & a_{32} & 0
\end{array}\right],
$$

the wave speed approaches zero as $\alpha$ is increased provided that

$$
a_{13}^{2}\left(a_{23}-a_{32}\right)=a_{31}^{2}\left(a_{21}-a_{12}\right) .
$$

The matrix (7.1) also illustrates the difference between the reaction system (1.1) and the reaction-diffusion system (1.2). Figure 7.2 shows trajectories for two values of $\alpha$. The dashed lines are the separatrices and these delineate the basins of attraction. We repeat that each pure strategy is an ESS and each has its own basin of attraction. Furthermore, Fig. 7.2 shows that the areas of these basins are comparable in size. When there are only two strategies and each is an ESS, then the direction of the travelling wave which joins these two equilibria is simply determined by which ESS has the larger basin of attraction. It appears unlikely that there is any comparably simple criterion when there are more than two strategies.

It is remarkable how strategy 2 facilitates the invasion of 3 . This demonstrates that short-time observations can be quite misleading. Population crashes may sometimes be very difficult to foresee. 

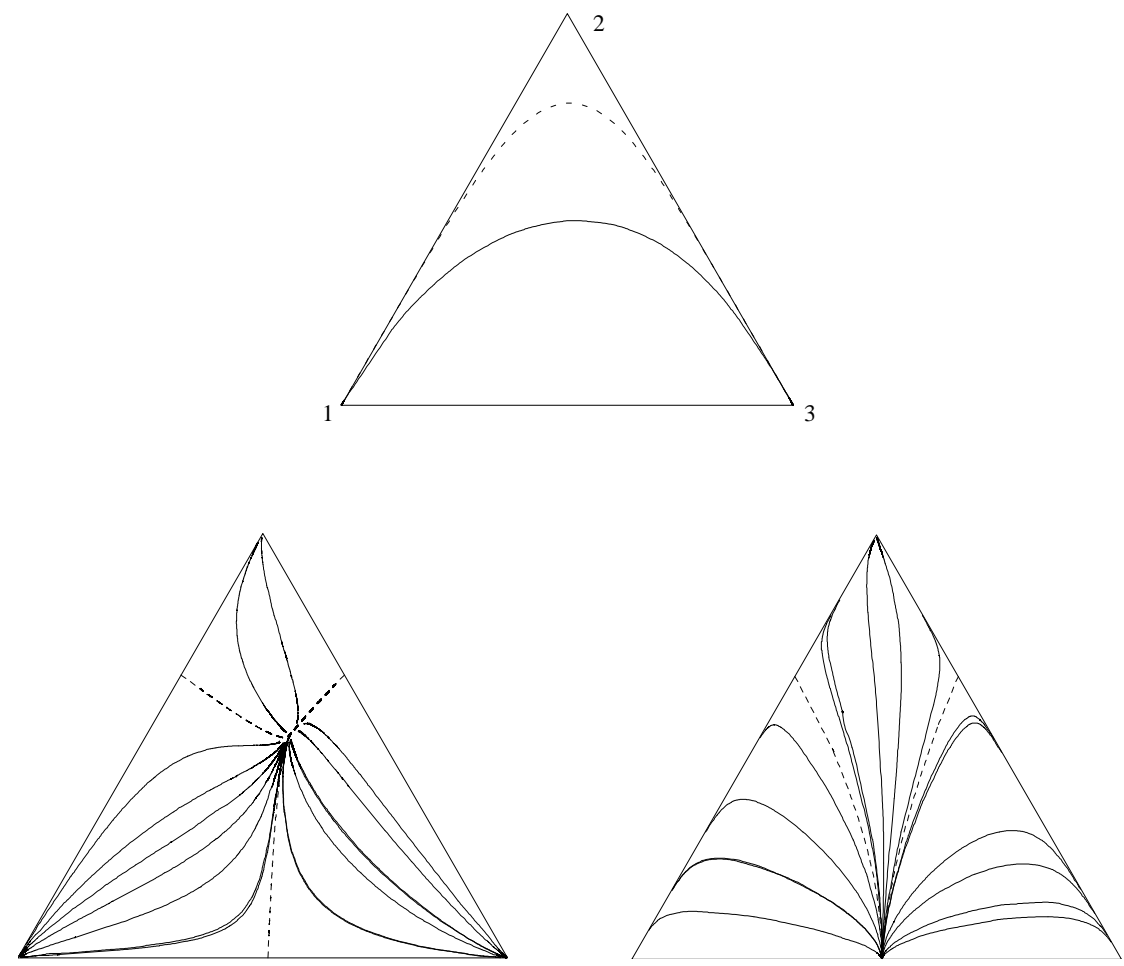

FIG. 7.2. Trajectories for the reaction system when $\alpha$ is 0.2 and 1.0

Appendix A. Condition for zero wave speed. We find the condition upon the entries of matrix (7.2) for the wave speed to approach zero as $\alpha \rightarrow \infty$. The argument that is given is suggestive only; no attempt is made to give a rigorous justification of the approximations used.

When $\alpha$ is large, the solution for the wave has a projection on the triangle of reference which approaches the the edges 12 and 23 . For the 12 edge, set $p_{3}=0$ and $p_{1}=1-p_{2}$. Then equation (4.2) gives with $c=0$,

$$
u_{2}^{\prime \prime}=u_{2}\left(1-u_{2}\right)\left[a_{21}-\left(a_{12}+a_{21}\right) u_{2}\right] .
$$

This can be integrated and it gives the value of $u_{2}^{\prime}$ at $u_{2}=1$ to be

$$
s_{1}=\sqrt{\frac{a_{21}-a_{12}}{6}} .
$$

In deriving this, the boundary condition $u_{2}^{\prime}=0$ at $u_{2}=0$ has been used. Thus the values of $u_{1}^{\prime}, u_{2}^{\prime}$ and $u_{3}^{\prime}$ at $u_{2}=1$ are

$$
-s_{1}, \quad s_{1} \text { and } 0
$$

respectively. A similar argument for the 23 edge shows that the values of $u_{1}^{\prime}, u_{2}^{\prime}$ and $u_{3}^{\prime}$ at $u_{2}=1$ are

$$
0, \quad-s_{2} \text { and } s_{2}
$$


where

$$
s_{2}=\sqrt{\frac{a_{23}-a_{32}}{6}} .
$$

These two sets of values for the derivatives are reconciled by considering what happens in the neighbourhood of the vertex 2 , i.e. where $u_{1}$ and $u_{3}$ are both small. It is assumed that near this point the dominant terms are those in which there is a factor of $\alpha$. Thus

$$
\begin{aligned}
& u_{1}^{\prime \prime}=\alpha u_{1} u_{3} a_{13}, \\
& u_{2}^{\prime \prime}=-\alpha u_{1} u_{3}\left[a_{13}+a_{31}\right], \\
& u_{3}^{\prime \prime}=\alpha u_{1} u_{3} a_{31} .
\end{aligned}
$$

Hence the changes in $u_{1}^{\prime}$ and $u_{3}^{\prime}$ are in the ratio $a_{13} / a_{31}$ which has to equal $s_{1} / s_{2}$. This gives the required condition as

$$
a_{13}^{2}\left(a_{23}-a_{32}\right)=a_{31}^{2}\left(a_{21}-a_{12}\right)
$$

Acknowledgements. We are very grateful to Josef Hofbauer (University of Vienna), Konstantin Mischaikow (Georgia Institute of Technology), Peter Polácik (Comenius University, Bratislava) and John Pym (University of Sheffield), for their valuable suggestions.

\section{REFERENCES}

[1] Chow, S.N. And Hale, J.K., Methods of Bifurcation Theory. Springer-Verlag, New York, (1982).

[2] Chow, S.N., Lin, X. And Mallet-Paret, J., Transition layers for singularly perturbed delay differential equations with monotone nonlinearities, J. Dyn. Diff. Eqns., 1 (1989), pp. 3-43.

[3] Conley, C. And Gardner, R., An application of the generalized Morse index to travelling wave solutions of a competitive reaction-diffusion model, Indiana J. Math., 33 (1984), pp. 319-365.

[4] Daners, D. And Koch Medina P., Abstract Evolution Equations, Periodic Problems and Applications. Pitman research Notes in Mathematics, vol. 279. Longmans, Harlow, (1992).

[5] Dunford, N. And Schwartz, J.T., Linear Operators, Part II: Spectral Theory, Interscience, New York, (1963).

[6] Ellison, G., Learning, local interaction and coordination, Econometrica, 61 (1993), pp. 10471071.

[7] Fife, P., Mathematical Aspects of Reacting and Diffusing Systems, Lect. Notes Biomath., vol. 28. Springer, Berlin, (1979).

[8] Fisher, R.A., The wave of advance of advantageous genes, Ann. Eug., 7 (1937), pp. 353-369.

[9] Henry, D., Geometric Theory of Semilinear Parabolic Equations, Lect. Notes in Math., vol. 840 Springer-Verlag, Berlin, (1980).

[10] Hofbauer, J., The spatially dominant equilibrium of a game, Ann. Oper. Res., 89 (1999), pp. $233-251$.

[11] Hofbauer, J., Hutson, V. And Vickers, G.T., Travelling waves for games in economics and biology, Nonlinear Anal. TMA, 32 (1997), pp. 1235-1244.

[12] Hutson, V., Stability in a reaction model of mutualism, SIAM J. Math. Anal., 17 (1986), pp. $58-66$.

[13] Hutson, V. And Vickers, G.T., Travelling waves and dominance of ESS's, J. Math. Biol., 30 (1992), pp. 457-471.

[14] Maynard Smith, J., The theory of games and the evolution of animal conflicts, J. theor. Biol., 47 (1974), pp. 209-221.

[15] Maynard Smith, J. And Price, G.R., The logic of animal conflict, Nature, 246 (1973), pp. $15-18$.

[16] Protter, M. And Weinberger, H., Maximum Principles in Differential Equations, Springer, New York, (1984).

[17] Robinson, C., Dynamical Systems, CRC Press, Boca Raton, (1995). 
[18] Tayler, P.D. And Jonker, L.B., Evolutionarily stable strategies and game dynamics, Math. Biosc., 40 (1978), pp. 145-156.

[19] Volpert, A.I., Volpert, V.A. And Volpert, V.A., Traveling Wave Solutions of Parabolic Systems, AMS Translations, vol. 140. (1991), Providence R.I.

[20] Von Neumann, J. And Morgenstern, O., Theory of Games and Economic Behaviour, Princeton Univ. Press, Princeton, (1944).

[21] Zeeman, E.C., Population dynamics from game theory, in Global Theory of Dynamical Systems. Lect. Notes in Math., vol. 819 (1980), eds. A. Nitecki and C. Robinson pp. 471-497. Springer, Berlin. 
